\title{
Sliding Mode LoAd Frequency Regulator FOR Different-Area PoWer Systems With COMMUNiCATION DELAYS
}

\author{
Bao Hoan LAI $I^{1}$, Anh-Tuan TRAN ${ }^{1}$, Van Van HUYNH ${ }^{2, *}$, Nhat Truong PHAM $M^{1,3}$ \\ ${ }^{1}$ Faculty of Electrical \& Electronics Engineering, Ton Duc Thang University, Ho Chi Minh City, \\ Vietnam \\ ${ }^{2}$ Modeling Evolutionary Algorithms Simulation and Artificial Intelligence, Faculty of Electrical \& \\ Electronics Engineering, Ton Duc Thang University, Ho Chi Minh City, Vietnam \\ ${ }^{3}$ Division of Computational Mechatronics, Institute for Computational Science, \\ Ton Duc Thang University, Ho Chi Minh City, Vietnam \\ *Corresponding Author: Van Van HUYNH (Email: huynhvanvan@tdtu.edu.vn) \\ (Received: 5-Mar-2021; accepted: 11-May-2021; published: 30-Jun-2021) \\ DOI: http://dx.doi.org/10.25073/jaec.202152.323
}

\begin{abstract}
In this article, a sliding mode control $(S M C)$ is proposed to deal with the frequency deviation problem in interconnected time-delay power systems (ITDPS) with two source power generations. First, the proportional and integral switching surface is used for each area to guarantee the frequency deviation reach zero in normal operating conditions. Then, the stability of the system is ensured with a new Linear Matrix Inequality (LMI) via Lyapunov stability theory. In addition, the SMC law is designed to guarantee the finite time reachability of the system. Finally, impacts of certain physical constraints affecting dynamic performance of the power network such as time-delay is proposed to consider the signal delay in the controller. Effectiveness of the suggested method is validated by simulation studies on the load frequency control under time-delays in the two-area, the step load disturbance and the mismatched uncertainty.
\end{abstract}

\section{Keywords}

Sliding mode control, load frequency control, multi-area power system.

\section{Introduction}

Load frequency control (LFC) is one of the vital and challenging problems in a power network. In a multi-area power system (MAPS), LFC has a significant role in terms of operating in the different structures and complex MAPS. The goals of the LFC are to keep the tie-line power and frequency interchanging in the MAPS at the desired values. In the past decades, researchers have applied both traditional and modern techniques to solve LFC problem [1-5].

In traditional approaches, PI and PID controllers are classical control methods that have been first designed to solve the LFC problem. They are very simple to design a structure for the PID controller in the power system by tuning the parameters. Sexana and Hote proposed a robust PID control method for the LFC problem in MAPS with different turbine devices by using Kharitonov's theorem [1]. The tuning method was proposed for PID-based LFC in the MAPS based on the internal model control method [6]. This approach aimed to attenuate the affection of the disturbance for improving the performance of the ITDPS. To decrease the required 
control effort and guarantee the settling time, overshoot, an optimal PID control method was designed based on LMI for both single power system and MAPS [7].

However, the PID controller has a limitation with the complex and non-linear power system because the PID controller is always considered constant parameters for a fixed structure in the power system. In addition, PID cannot achieve an expectation performance when the disturbance is applied in the system. Therefore, the combination of intelligent control and optimization technique has been proposed to choose the optimal parameters for the PID controller to improve the system stability and performance in the LFC of the MAPS. The method given in [8] proposed a robust PID control method for LFC by applying the imperialist competitive algorithm to reduce the disturbance affection. Rejesh and Dash proposed an improvement sine and cosine algorithm to tune the parameters for a PID-based fuzzy logic (FL) controller for the LFC of the ITDPS [9]. To handle the load disturbance and the system parameter uncertainty, some studies designed a fractional order-based PID for the LFC of the power network $[10,11]$.

In modern control approaches, some advanced control methods have been suggested to handle the LFC problem. Cuckoo search and optimization method were proposed for the LFC in the power network to enhance the system performance in case of various loading conditions $[12$, 13]. The method given in [14] proposed a decentralized control approach based on the Lyapunov theory for the LFC of MAPS in case of boundary disturbance. Yousef et al. designed an adaptive FLC with direct and indirect schemes for the LFC of MAPS in case of having unknown parameters [15]. Moreover, a robust control was deployed for the LFC of the MAPS given in [16, 17] to satisfy the robust performance with the load variation and guarantee the internal stability of the ITDPS.

Sliding mode control (SMC) method is nonlinear and variable structure control method. SMC has been applied in many different fields, such as induction motor drives [18], micro-grid [19], converter [20], vehicle systems [21, 22], interconnected electrical systems [23, 24], and robotics [25]. Since the characteristics of the components in almost all systems are also nonlinear, it is suitable to improve the system stability and performance by applying SMC and its relevant control methods. Besides, SMC is wellknown as one of the modern control methods that have been investigated to resolve the LFC of the MAPS in the past decade. In [26], a decentralized SMC method is designed for the LFC of the MAPS with wind turbine under matched and mismatched uncertainties. However, the integral sliding surface gives low accuracy due to chattering phenomenon. Therefore, Le Ngoc Minh et al. proposed a double integral sliding surface based on the decentralized adaptive control to solve the above problem [27]. Moreover, researchers have tried to combine intelligent techniques with SMC to design the optimal and robust control for the LFC of the MAPS. $\mathrm{Mu}$ et al. combined adaptive learning and SMC for the LFC of the power network to improve the system performance under load disturbances and parameter uncertainties [28]. Guo and Liu proposed an SMC method based on PSO for the LFC in the MAPS that helped to find the optimal values for transferring vector and feedback gains, and hence, reduce overshoot [29].

Practically, in the real power system, the time-delay or the communication delay is focused on investigating the LFC problem in the MAPS. Mi et al. designed a novel SMC based on decentralizing control to ensure the system stability under time-delay for the LFC of the MAPS with wind power integration [30]. Onyeka et al. designed a robust decentralized LFC for the MAPS under the time-delay effect by using the SMC technique with Lyapunov function to manage the frequency deviation and power interchange [31]. Sun et al. developed a new sliding surface for designing SMC law which is robust stability in terms of LMI for the LFC of the MAPS with time-delay [32].

In this article, the robustness of integral sliding surface is designed for the LFC of the MAPS with two-source power generation under communication delay. Our main contribution is presented as follows: 
- The sliding mode controller is suggested for different area power network with thermal and hydro power plants under communication delay.

- The stability of the ITDPS is ensured based on the Lyapunov theory with the new LMI approach.

- Experimental simulation results indicate that the system performance is better than recently researches in terms of overshoot and settling time.

\section{Mathematical model of interconnected time-delay power system (ITDPS)}

Schematic model of the ITDPS with the thermal power station in the first area and the hydro power station in second area is shown as Fig. 1.

From Fig. 1, in the dynamic equations of the above power network may be expressed as following

$$
\begin{aligned}
\Delta \dot{f}_{1}(t)= & -\frac{1}{T_{p 1}} \Delta f_{1}(t)+\frac{K_{p 1}}{T_{p 1}} \Delta P_{m 1}(t) \\
& -\frac{K_{p 1}}{T_{p 1}} \Delta P_{t i e 1,2}(t)-\frac{K_{p 1}}{T_{p 1}} \Delta P_{d_{1}} \\
\Delta \dot{P}_{m 1}(t)= & \left(\frac{1}{T_{r 1}}-\frac{K_{r 1}}{T_{t h 1}}\right) \Delta P_{t h 1}(t) \\
& +\frac{K_{r 1}}{T_{t h 1}} \Delta P_{v 1}(t)-\frac{1}{T_{r 1}} \Delta P_{m 1}(t) \\
\Delta \dot{P}_{t h 1}(t)= & \frac{1}{T_{t h 1}} \Delta P_{v 1}(t)-\frac{1}{T_{t h 1}} \Delta P_{t h 1}(t) \\
\Delta \dot{P}_{v 1}(t)= & -\frac{1}{T_{g 1} R_{1}} \Delta f_{1}(t)-\frac{1}{T_{g 1}} \Delta P_{v 1}(t) \\
& -\frac{1}{T_{g 1}} \Delta E_{1}(t-\psi)+\frac{1}{T_{g 1}} \Delta P_{r e f 1}(t) \\
\Delta \dot{f}_{2}(t)= & -\frac{1}{T_{p 2}} \Delta f_{2}(t)+\frac{K_{p 2}}{T_{p 2}} \Delta P_{m 2}(t) \\
& -a_{12} \frac{K_{p 2}}{T_{p 2}} \Delta P_{t i e 1,2}(t)-\frac{K_{p 2}}{T_{p 2}} \Delta P_{d_{2}}(t)
\end{aligned}
$$

$$
\begin{aligned}
\Delta P_{m 2}(t)= & \left(\frac{2 T_{r s 2}}{T_{r h 2} T_{g 2}}-\frac{2}{T_{r h 2}}\right) \Delta P_{g 2}(t) \\
& +\left(\frac{2}{T_{w 2}}+\frac{2}{T_{r h 2}}\right) \Delta P_{v 2}(t) \\
& -\frac{2}{T_{w 2}} \Delta P_{m 2}(t)+\frac{2 T_{r s 2}}{T_{r h 2} T_{g 2} R_{2 i}} \Delta f_{2}(t) \\
& -\frac{2 T_{r s 2}}{T_{r h 2} T_{g 2}} \Delta P_{r e f 2}(t)
\end{aligned}
$$

$$
\begin{aligned}
\Delta P_{v 2}= & \frac{-T_{r s 2}}{T_{r h 2} T_{h 2} R_{2 i}} \Delta f_{2}(t) \\
& +\left(\frac{1}{T_{r h 2}}-\frac{T_{r s 2}}{T_{r h 2} T_{g 2}}\right) \Delta P_{g 2}(t) \\
& +\frac{-1}{T_{r h 2}} \Delta P_{v 2}(t)+\frac{T_{r s 2}}{T_{r h 2} T_{g 2}} \Delta P_{r e f 2}(t)
\end{aligned}
$$

$$
\begin{aligned}
\Delta \dot{P}_{g 2}(t)= & -\frac{1}{T_{g 2} R_{2}} \Delta f_{2}(t)-\frac{1}{T_{g 2}} \Delta P_{g 2}(t) \\
& -\frac{1}{T_{g 2}} \Delta E_{2}(t-\varsigma)+\frac{1}{T_{g 2}} \Delta P_{r e f 2}(t)
\end{aligned}
$$

$$
\begin{gathered}
\Delta \dot{P}_{t i e 1,2}(t)=2 \pi T_{12}\left[\Delta f_{1}(t)-\Delta f_{2}(t)\right] \\
\Delta \dot{E}_{1}(t)=K_{B 1} \Delta f_{1}(t)+\Delta P_{t i e 1,2}(t) \\
\Delta \dot{E}_{2}(t)=K_{B 2} \Delta f_{2}(t)+a_{12} \Delta P_{t i e 1,2}(t)
\end{gathered}
$$

All parameters and symbols of the above dynamic equations (1)-(11) are defined in the Appendix A. So, the two-area interconnected electricity network with communication delays is given by Fig. 1 which can be rewritten in the state-space representation as below

$$
\begin{aligned}
\dot{z}(t)= & A z(t)+D z(t-\psi)+T z(t-\varsigma) \\
& +B u(t)+F \Delta P_{D}(t) \\
n(t)= & C z(t)
\end{aligned}
$$

where the state vector is denoted as below

$$
\begin{aligned}
& z(t)=\left[\begin{array}{ccc}
\Delta F_{1}(t) & \Delta P_{m 1}(t) & \Delta P_{t h 1}(t)
\end{array}\right. \\
& \Delta P_{v 1}(t) \quad \Delta F_{2}(t) \quad \Delta P_{m 2}(t) \\
& \Delta P_{v 2}(t) \quad \Delta P_{g 2}(t) \quad \Delta P_{t i e, 1}(t) \\
& \Delta E_{1}(t) \quad \Delta E_{2}(t)
\end{aligned}
$$




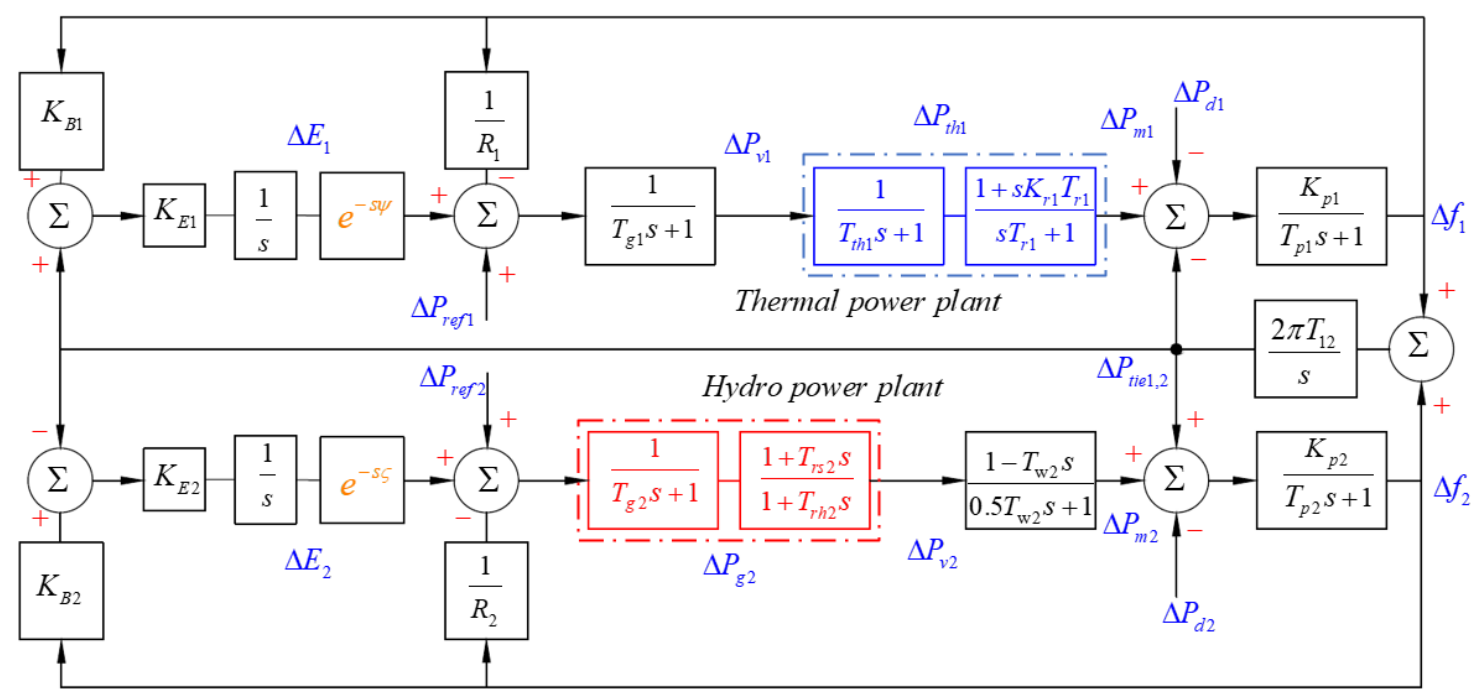

Fig. 1: The structure of the different-area LFC model in a power network with time-delay.

and the system matrices $A, B, F, D, T$ are given as below

$D=\left[\begin{array}{ccccccccccc}0 & 0 & 0 & 0 & 0 & 0 & 0 & 0 & 0 & 0 & 0 \\ 0 & 0 & 0 & 0 & 0 & 0 & 0 & 0 & 0 & 0 & 0 \\ 0 & 0 & 0 & 0 & 0 & 0 & 0 & 0 & 0 & 0 & 0 \\ 0 & 0 & 0 & 0 & \frac{-1}{T_{g 1}} & 0 & 0 & 0 & 0 & 0 & 0 \\ 0 & 0 & 0 & 0 & 0 & 0 & 0 & 0 & 0 & 0 & 0 \\ 0 & 0 & 0 & 0 & 0 & 0 & 0 & 0 & 0 & 0 & 0 \\ 0 & 0 & 0 & 0 & 0 & 0 & 0 & 0 & 0 & 0 & 0 \\ 0 & 0 & 0 & 0 & 0 & 0 & 0 & 0 & 0 & 0 & 0 \\ 0 & 0 & 0 & 0 & 0 & 0 & 0 & 0 & 0 & 0 & 0 \\ 0 & 0 & 0 & 0 & 0 & 0 & 0 & 0 & 0 & 0 & 0 \\ 0 & 0 & 0 & 0 & 0 & 0 & 0 & 0 & 0 & 0 & 0\end{array}\right]$,

$$
B=\left[\begin{array}{cc}
0 & 0 \\
0 & 0 \\
0 & 0 \\
\frac{1}{T_{g 1}} & 0 \\
0 & 0 \\
0 & \frac{-2 T_{r s 2}}{T_{r h 2} T_{g 2}} \\
0 & \frac{T_{r s 2}}{T_{r h 2} T_{g 2}} \\
0 & \frac{1}{T_{g 2}} \\
0 & 0 \\
0 & 0 \\
0 & 0
\end{array}\right]
$$

$T=\left[\begin{array}{ccccccccccc}0 & 0 & 0 & 0 & 0 & 0 & 0 & 0 & 0 & 0 & 0 \\ 0 & 0 & 0 & 0 & 0 & 0 & 0 & 0 & 0 & 0 & 0 \\ 0 & 0 & 0 & 0 & 0 & 0 & 0 & 0 & 0 & 0 & 0 \\ 0 & 0 & 0 & 0 & 0 & 0 & 0 & 0 & 0 & 0 & 0 \\ 0 & 0 & 0 & 0 & 0 & 0 & 0 & 0 & 0 & 0 & 0 \\ 0 & 0 & 0 & 0 & 0 & 0 & 0 & 0 & 0 & 0 & 0 \\ 0 & 0 & 0 & 0 & 0 & 0 & 0 & 0 & 0 & 0 & 0 \\ 0 & 0 & 0 & 0 & 0 & 0 & 0 & 0 & \frac{-1}{T_{g 2}} & 0 & 0 \\ 0 & 0 & 0 & 0 & 0 & 0 & 0 & 0 & 0 & 0 & 0 \\ 0 & 0 & 0 & 0 & 0 & 0 & 0 & 0 & 0 & 0 & 0 \\ 0 & 0 & 0 & 0 & 0 & 0 & 0 & 0 & 0 & 0 & 0\end{array}\right]$,

$F=\left[\begin{array}{cc}\frac{-K_{P 1}}{T_{p 1}} & 0 \\ 0 & 0 \\ 0 & 0 \\ 0 & 0 \\ 0 & \frac{-K_{P 2}}{T_{p 2}} \\ 0 & 0 \\ 0 & 0 \\ 0 & 0 \\ 0 & 0 \\ 0 & 0 \\ 0 & 0\end{array}\right]$ 


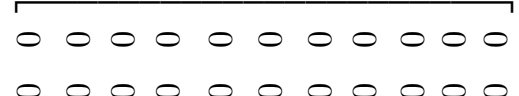

00000000000

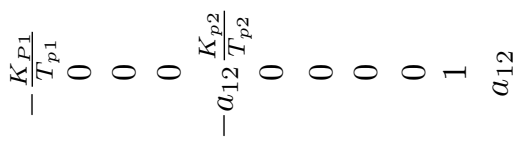

000000

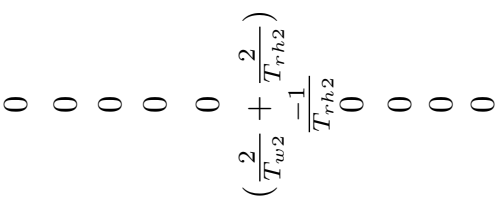

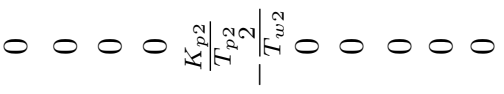

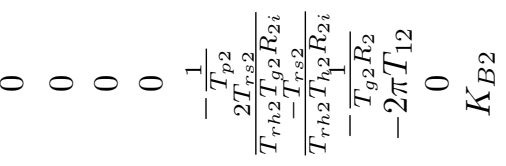

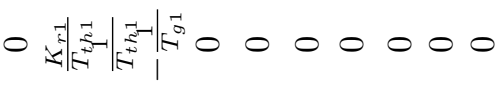

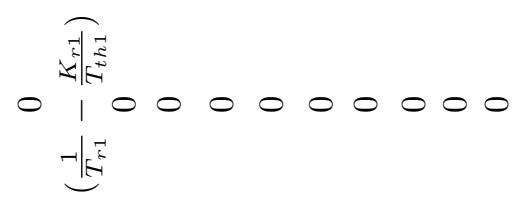

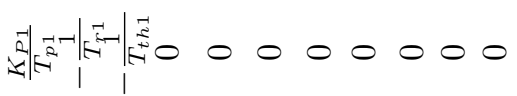

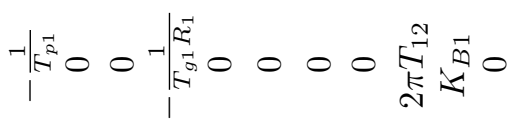
11
In addition, by considering time-delay communication issues, so the dynamic model of the ITDPS in Eq. (12) is re-written as

$$
\begin{aligned}
\dot{z}(t)= & {[A+\Delta A(z, t)] z(t) } \\
& +[D+\Delta D(z, t-\psi)] z(t-\psi) \\
& +[T+\Delta T(z, t-\varsigma)] z(t-\varsigma) \\
& +B[u(t)+\xi(z, t)]+F \Delta P_{D}(t) \\
= & A z(t)+D z(t-\tau) \\
& +T z(t-\varsigma)+B u(t)+w(z, t) \\
n(t)= & C z(t)
\end{aligned}
$$

where $A, B, D, T, F$ is the nominal values, the unknown matrices $\Delta A(z, t), \Delta D(z, t-\tau)$ and $\Delta T(z, t-\varsigma)$ are denoted by their time variant system of parametric variations and $B \xi(z, t)$ is the disturbance input signal; $w(z, t)$ is called the lumped uncertainties which can be estimated and we can define it as the following

$$
\begin{aligned}
w(z, t)= & \Delta A(z, t) z(t)+\Delta D(z, t-\psi) z(t-\psi) \\
& +\Delta T(z, t-\varsigma) z(t-\varsigma) \\
& +B \xi(z, t)+F \Delta P_{D}(t)
\end{aligned}
$$

Remark 1: In the real power network, the time delay usually exists in sending a plant signal between the sensor and the controller $\psi_{1}$ and in sending control signals between the controller and LFC system $\psi_{2}$ [37]. In this approach, we combine the two above time delay as a single delay component $\psi=\psi_{1}+\psi_{2}$ for each area which is the same with the method given in [37]. However, the time delay of each area has differential value which is more general than the one in [37].

In the best possible way, some assumptions are indicated to describe the reality and feasibility of the power system as well as the system parameters based LFC strategies under certain conditions.

Assumption 1: The lumped uncertainties $w(z, t)$, which includes time-delay in the state matrix and the differential of $\dot{w}(z, t)$ is bounded. i.e., there exist the known scalars $\gamma$ and $\bar{\gamma}$ such that $\|w(z, t)\| \leq \gamma$ and $\|\dot{w}(z, t)\| \leq \bar{\gamma}$, where $\|\cdot\|$ is matrix norm.

Assumption 2: The time delay state vector must be satisfied the conditions

$\|z(t-\tau)\| \leq z_{\max }$ and $\|z(t-\varsigma)\| \leq z_{\max }$, $z_{\max }=\max \|z\|$. 
In addition, some lemmas are recalled for proving the system stability as given in [33] and [34]:

Lemma 1 [33]: Let $X$ and $Y$ are actual matrices with appropriate dimension then, for any scalar $\mu>0$, the sequent matrix inequality obtains

$$
X^{T} Y+Y^{T} X \leq \mu X^{T} X+\mu^{-1} Y^{T} Y .
$$

Lemma 2 [34]: If the matrix:

$$
\left[\begin{array}{cc}
P(z) & \Gamma(z) \\
\Gamma^{T}(z) & Q(z)
\end{array}\right]>0
$$

where $P(z)=P^{T}(z), Q(z)=Q^{T}(z)$ and $\Gamma(z)$ which depends affinitively on $z$. Therefore, $Q(z)>0$ and $P(z)-\Gamma(z) Q^{-1}(z) \Gamma^{T}(z)>0$.

Lemma 3 [34]: Assume that $z \in R^{n}, y \in R^{n}$, $M \in R^{n \times n}, M$ is the positive definite matrix. Then, the inequality

$$
z^{T} M y+y^{T} M z \leq \frac{1}{\varepsilon} z^{T} M z+\varepsilon y^{T} M y
$$

holds for all $\varepsilon>0$.

\section{Proposed sliding mode controller design with time-delay}

In this approach, an integral SMC method is suggested and improved for the MAPS (13) with parametric disturbances and uncertainties.

$$
\sigma[z(t)]=N z(t)-\int_{0}^{t} N(A-B K) z(\tau) \mathrm{d} \tau
$$

where the matrix $N$ is chosen to ensure that matrix $N B$ is non-singular. Matrix $K$ is achieved via pole assignment so that the eigenvalues of matrix $(A-B K)$ are smaller than zero.

If we recognize and differentiate $\sigma[z(t)]$ related to time combined with (13), then

$$
\begin{aligned}
\dot{\sigma}[z(t)]= & N[A z(t)+D z(t-\psi)+T z(t-\varsigma) \\
& +B u(t)+w(z, t)]-N(A-B K) z(t)
\end{aligned}
$$

So, the setting $\sigma[z(t)]=\dot{\sigma}[z(t)]=0$, the control is re-written by

$$
\begin{aligned}
u_{e q}(t)= & -(N B)^{-1}[N A z(t)+N D z(t-\psi) \\
& +N T z(t-\varsigma)+N w(z, t)] \\
& -N(A-B K) z(t)
\end{aligned}
$$

To satisfy the reaching condition, the design controller becomes

$$
u(t)=u_{e q}(t)-\delta \operatorname{sgn}(\sigma[z(t)])
$$

where $u(t)$ is the proposed controller for MAPS. Then controller is further rewritten as

$$
\begin{aligned}
u(t)= & -(N B)^{-1}[N B K z(t)+N \mathrm{D} z(t-\psi) \\
& +N T z(t-\varsigma)+N w(z, t)] \\
& -\delta \operatorname{sgn}(\sigma[z(t)])
\end{aligned}
$$

As studied in SMC theory, to determine the dynamic equation on the ISS, we make $u(t)=$ $u_{e q}(t)$ and substitute into Eq. (13), we get

$$
\begin{aligned}
\dot{z}(t)= & (A-B K) z(t) \\
& +\left[I-B(N B)^{-1} N\right] D z(t-\psi) \\
& +\left[I-B(N B)^{-1} N\right] T z(t-\varsigma) \\
& +\left[I-B(N B)^{-1} N\right] w(z, t)
\end{aligned}
$$

Then, the dynamic equation (23) of sliding mode is asymptotically stable based on below Theorem.

Theorem 1: The sliding mode dynamic equation (23) is asymptotically stable if and only if there includes the symmetric positive definite matrices $Q, P$, Rand positive scalars $\chi$ and $q$ as the following LMIs hold

[Eq. (24) on the next page]

where $\prod=(A-B K)^{T} Q+Q(A-B K)+2 Q+$ $P+R$.

Proof 1: To research and analyze the stability of the sliding motion (23), the Lyapunov function is selected as below

$$
\begin{aligned}
V=z^{T}(t) Q z(t) & +\int_{t-\psi}^{t} z^{T}(s) P z(s) \mathrm{d} s \\
& +\int_{t-\varsigma}^{t} z^{T}(s) R z(s) \mathrm{d} s
\end{aligned}
$$

where $Q, P, R>0$ satisfies Eq. (25). Then, taking the time derivative of Eq. (25) and using 


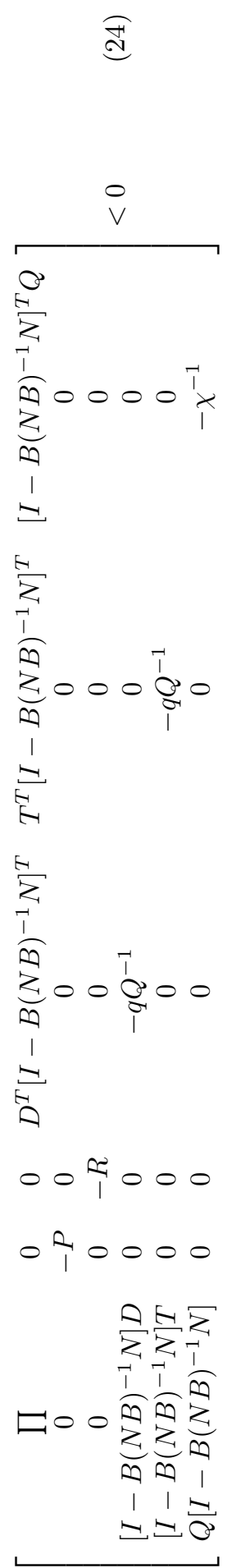

Eq. (23), we have

$$
\begin{aligned}
\dot{V}= & {\left[z^{T}(t)\left[(A-B K)^{T} Q+Q(A-B K)\right] z(t)\right.} \\
& +z^{T}(t-\psi) D^{T}\left[I-B(N B)^{-1} N\right]^{T} Q z(t) \\
& +z^{T}(t) Q\left[I-B(N B)^{-1} N\right] D z(t-\psi) \\
& +z^{T}(t-\varsigma) T^{T}\left[I-B(N B)^{-1} N\right]^{T} Q z(t) \\
& +z^{T}(t) Q\left[I-B(N B)^{-1} N\right] T z(t-\varsigma) \\
& +w^{T}(z, t)\left[I-B(N B)^{-1} N\right]^{T} Q z(t) \\
& \left.+z^{T}(t) Q\left[I-B(N B)^{-1} N\right] w(z, t)\right] \\
& +z^{T}(t) P z(t)-z^{T}(t-\psi) P z(t-\psi) \\
& +z^{T}(t) R z(t)-z^{T}(t-\varsigma) R z(t-\varsigma) \quad(26)
\end{aligned}
$$

To apply the Lemma 1 to Eq. (26), we have

$$
\begin{aligned}
\dot{V} \leq & z^{T}(t)\left[(A-B K)^{T} Q+Q(A-B K)\right] z(t) \\
& +z^{T}(t-\psi) D^{T}\left[I-B(N B)^{-1} N\right]^{T} Q z(t) \\
& +z^{T}(t) Q\left[I-B(N B)^{-1} N\right] D z(t-\psi) \\
& +z^{T}(t-\varsigma) T^{T}\left[I-B(N B)^{-1} N\right]^{T} Q z(t) \\
& +z^{T}(t) Q\left[I-B(N B)^{-1} N\right] T z(t-\varsigma) \\
& +\chi^{-1} z^{T}(t) Q\left[I-B(N B)^{-1} N\right] \\
& \times\left[I-B(N B)^{-1} N\right]^{T} Q z(t) \\
& +\chi w^{T}(z, t) w(z, t) \\
& +z^{T}(t) P z(t)-z^{T}(t-\psi) P z(t-\psi) \\
& +z^{T}(t) R z(t)-z^{T}(t-\varsigma) R z(t-\varsigma) \quad(27
\end{aligned}
$$

Combining Lemma 3 and Eq. (27), we obtain

$$
\begin{aligned}
\dot{V} \leq & z^{T}(t)\left[(A-B K)^{T} Q+Q(A-B K)\right] z(t) \\
& +z^{T}(t-\psi) D^{T}\left[I-B(N B)^{-1} N\right]^{T} \\
& \times Q\left[I-B(N B)^{-1} N\right] D z(t-\psi) \\
& +z^{T}(t-\varsigma) T^{T}\left[I-B(N B)^{-1} N\right]^{T} \\
& \times Q\left[I-B(N B)^{-1} N\right] T z(t-\varsigma) \\
& +\chi^{-1} z^{T}(t) Q\left[I-B(N B)^{-1} N\right] \\
& \times\left[I-B(N B)^{-1} N\right]^{T} Q z(t) \\
& +z^{T}(t) Q z(t)+z^{T}(t) Q z(t) \\
& +\chi w^{T}(z, t) w(z, t) \\
& +z^{T}(t) P z(t)-z^{T}(t-\psi) P z(t-\psi) \\
& +z^{T}(t) R z(t)-z^{T}(t-\varsigma) R z(t-\varsigma) \quad(28)
\end{aligned}
$$

The matrix $D^{T}\left[I-B(N B)^{-1} N\right]^{T} Q[I-$ $\left.B(N B)^{-1} N\right] D$ and $T^{T}\left[I-B(N B)^{-1} N\right]^{T} Q[I-$ $\left.B(N B)^{-1} N\right] T$ are semi-positive definite. Since 
the $z(t)$ is independent of each other. Then, from Eq. (31) of paper [35], the following is true

$$
V(z(t-\psi), z(t-\varsigma)) \leq q V(z(t))
$$

for $q>1$, is equivalent to

$$
\begin{aligned}
& z^{T}(t-\psi) D^{T}\left[I-B(N B)^{-1} N\right]^{T} \\
& \times Q\left[I-B(N B)^{-1} N\right] D z(t-\psi) \\
\leq & q z^{T}(t) D^{T}\left[I-B(N B)^{-1} N\right]^{T} \\
& \times Q\left[I-B(N B)^{-1} N\right] D z(t)
\end{aligned}
$$

and

$$
\begin{aligned}
& z^{T}(t-\varsigma) T^{T}\left[I-B(N B)^{-1} N\right]^{T} \\
& \times Q\left[I-B(N B)^{-1} N\right] T z(t-\varsigma) \\
\leq & q z^{T}(t) T^{T}\left[I-B(N B)^{-1} N\right]^{T} \\
& \times Q\left[I-B(N B)^{-1} N\right] T z(t)
\end{aligned}
$$

Then, the following equation can be achieved

$$
\begin{aligned}
\dot{V} \leq & z^{T}(t)\left[(A-B K)^{T} Q+Q(A-B K)\right. \\
& +q D^{T}\left[I-B(N B)^{-1} N\right]^{T} \\
& \times Q\left[I-B(N B)^{-1} N\right] D \\
& +q T^{T}\left[I-B(N B)^{-1} N\right]^{T} \\
& \times Q\left[I-B(N B)^{-1} N\right] T \\
& +\chi^{-1} Q\left[I-B(N B)^{-1} N\right] \\
& \times\left[I-B(N B)^{-1} N\right]^{T} Q \\
& +2 Q+P+R] z(t) \\
& -z^{T}(t-\psi) P z(t-\psi) \\
& -z^{T}(t-\varsigma) R z(t-\varsigma) \\
& +\chi_{i} w_{i}^{T}\left(z_{i}, t\right) w_{i}\left(z_{i}, t\right)
\end{aligned}
$$

Using the assumption 1, Eq. (32) becomes

$$
\begin{aligned}
\dot{V} \leq & z^{T}(t)\left[(A-B K)^{T} Q+Q(A-B K)\right. \\
& +q D^{T}\left[I-B(N B)^{-1} N\right]^{T} \\
& \times Q\left[I-B(N B)^{-1} N\right] D \\
& +q T^{T}\left[I-B(N B)^{-1} N\right]^{T} \\
& \times Q\left[I-B(N B)^{-1} N\right] T \\
& +\chi^{-1} Q\left[I-B(N B)^{-1} N\right] \\
& \times\left[I-B(N B)^{-1} N\right]^{T} Q \\
& +2 Q+P+R] z(t) \\
& -z^{T}(t-\psi) P z(t-\psi) \\
& -z^{T}(t-\varsigma) R z(t-\varsigma)+\iota
\end{aligned}
$$

where $\iota=\chi \gamma^{2}$.

Define the extended vector

$$
\Psi(t)=\left[\begin{array}{lll}
z^{T}(t) & z^{T}(t-\psi) & z^{T}(t-\varsigma)
\end{array}\right]^{T}
$$

and

$$
\dot{V} \leq \Psi^{T}(t) \Theta \Psi(t)+\iota
$$

Combining LMI (24) and Lemma 2, we obtain

$$
\Theta=-\left[\begin{array}{ccc}
\Xi & 0 & 0 \\
0 & -P & 0 \\
0 & 0 & -R
\end{array}\right]>0
$$

where

$$
\begin{aligned}
\Xi= & -\left[(A-B K)^{T} Q+Q(A-B K)\right. \\
& +q D^{T}\left[I-B(N B)^{-1} N\right]^{T} \\
& \times Q\left[I-B(N B)^{-1} N\right] D \\
& +q T^{T}\left[I-B(N B)^{-1} N\right]^{T} \\
& \times Q\left[I-B(N B)^{-1} N\right] T \\
& +\chi^{-1} Q\left[I-B(N B)^{-1} N\right] \\
& \times\left[I-B(N B)^{-1} N\right]^{T} Q \\
& +2 Q+P+R]>0
\end{aligned}
$$

Combining Eqs. (35) and (36), we have

$$
\dot{V}[z(t)] \leq-\lambda_{\min }(\Theta)\|\Psi(t)\|^{2}+\iota
$$

where the eigenvalue $\lambda_{\min }(\Theta)>0$ and the constant value $\iota$. Therefore, $\dot{V}<0$ is achieved with $\|z(t)\|>\sqrt{\frac{\iota}{\lambda_{\min }(\Theta)}}$. The sliding motion of system (23) is asymptotically stable.

If $\dot{\mathrm{V}}[z(t)] \leq 0$ shows that, the LMI (24) holds, therefore, it further explains that the system (13) is asymptotically stable. To prove the reachability of state variable $z(t)$, we again use Lyapunov stability theory. We assumed there exist $z(t)>0$, then Lyapunov function becomes

$$
V[z(t)]=\frac{1}{2} \sigma[z(t)]^{2}
$$

By taking the derivative of Eq. (39), we get

$$
\dot{V}[z(t)]=\sigma[z(t)]^{T} \dot{\sigma}[z(t)]
$$

Combining Eqs. (19) and (40), we obtain

$$
\begin{aligned}
\dot{V}[z(t)]= & \sigma[z(t)]^{T}\{N A z(t)+N B u(t) \\
& +N D z(t-\psi)+N T z(t-\varsigma)+N w(z, t) \\
& -N(A-B K) z(t)\}
\end{aligned}
$$


If we substitute $u(t)$ into Eq. (41), it is further simplified below

$$
\dot{V}[z(t)]=-\delta \operatorname{sgn}(\sigma[z(t)]) \sigma[z(t)]^{T}
$$

Therefore, Eq. (42) shows that $\dot{\mathrm{V}}[z(t)]<0$ which demonstrates the reachability of the system states to the sliding surface.

Remark 2: The recent SMC method is applied for the ITDPS which can be seen in paper [38]. However, the approach given in [38] considers only single time delay in the system dynamic equation with only one kind of power plant. In this approach, the ITDPS is considered with different kind of power plant for each area and the system dynamic equation (13) has two kinds of time delay and different value of the time delay for each area. Therefore, the proposed approach is a better choice for the LFC of real ITDPS.

Remark 3: The new LFC method for the MAPS is given by [39]. The approach given in [39] is achieved under the assumptions that there is not time delay in the MAPS and there is only one kind of power plant. Therefore, in the proposed approach, the two above limitations have been solved.

\section{Results and discussions}

Based on the two-area ITDPS where thermal and hydro power stations in first and second areas, respectively. Three cases are presented to demonstrate the power of the suggested SMC under load disturbances and mismatched uncertainties. A conventional two-area power network (TAPN) is analyzed for the robustness and effectiveness of the suggested integral SMC. The parameters of TAPN were presented in [36] as display in Appendix B.

Case 1: The nominal value is used for the system parameters. The step load disturbances set as the same [36] with value at the first area is $0.007 \mathrm{pu}$ at $t=1 \mathrm{~s}$ and the second area is 0.01 $\mathrm{pu}$ at $t=3 \mathrm{~s}$. The constant delays of $\psi=0.1 \mathrm{~s}$ in the first area and $\varsigma=0.2 \mathrm{~s}$ in the second area are considered in this simulation. The results of frequency variation in both areas $\Delta f_{1}, \Delta f_{2}$ with the proposed controller are displayed in Fig. 2. The tie-line power deviation $\Delta P_{\text {tie }}$ is displayed in Fig. 3. The test results are based on frequency and tie-line deviation of proposed integral SMC is compared with [36].

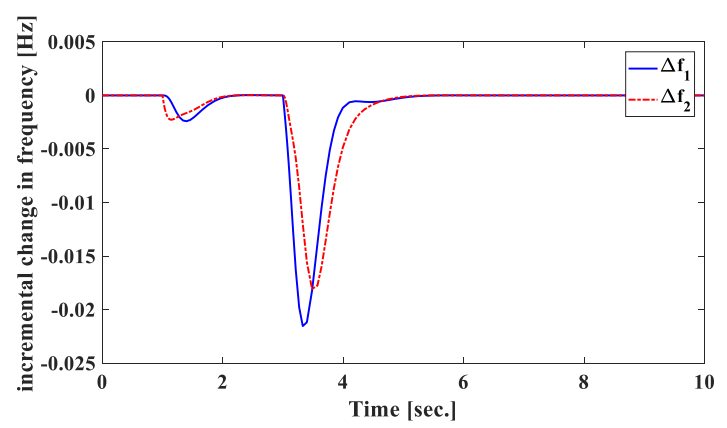

Fig. 2: Frequency variation.

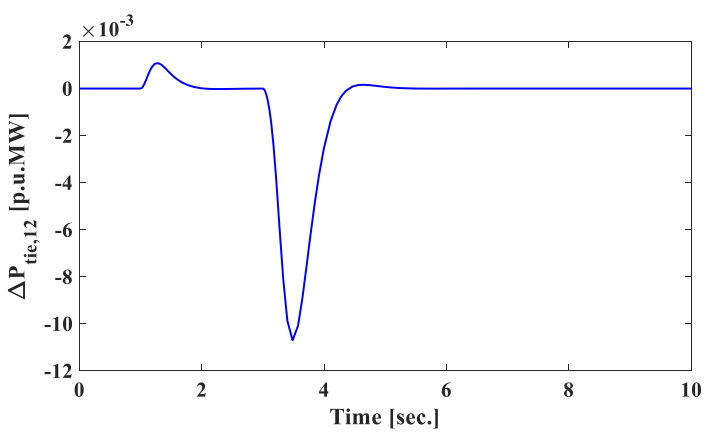

Fig. 3: Tie-line variation.

Remark 4: In this case, the suggested controller is still good performance in term of smaller over shoot and faster settling time even though the influence of communication delay is considered in different areas power network in comparing with [36]. Clearly, the proposed control approach is powerful and useful to control ITDPS under step load disturbance.

Case 2: The ITDPS is simulated under random load disturbances at every $6 \mathrm{~s}$ as shown in Fig. 4. The maximum frequency variation $\Delta f_{1}, \Delta f_{2}$ of two areas under time-delay the same with the previous case are shown in Fig. 5 with values of $0.06 \mathrm{~Hz}$ and $0.07 \mathrm{~Hz}$, respectively. The maximum tie-line variation $\Delta P_{\mathrm{tie} 1,2}$ between the first area and the second area is displayed in Fig. 6 with value of $0.031 \mathrm{pu}$. The proposed method has significantly improved the performance of frequency stability in the ITDPS. 


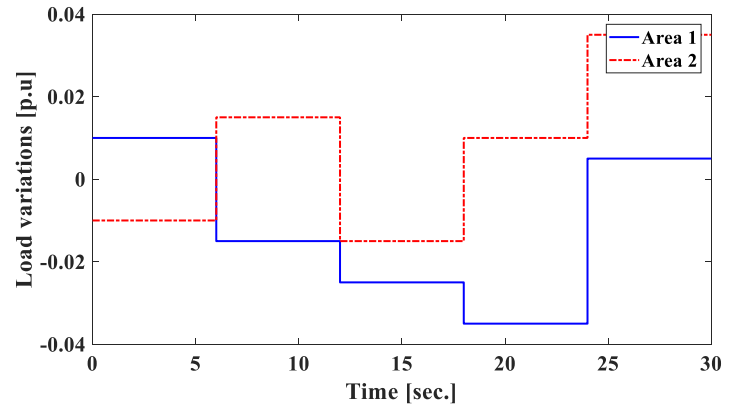

Fig. 4: Load variations.

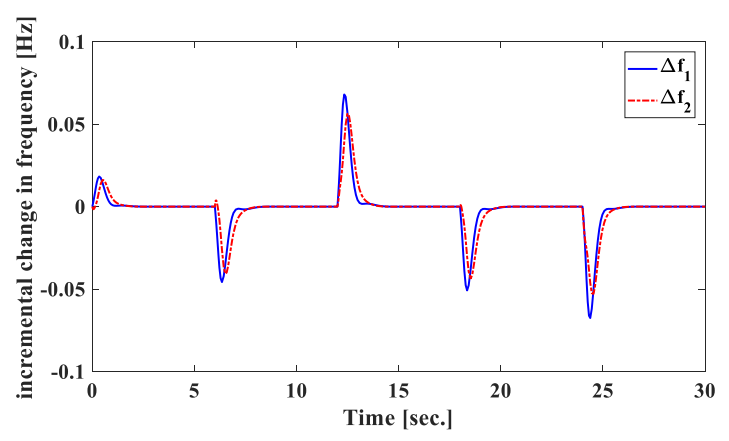

Fig. 5: Frequency variation.

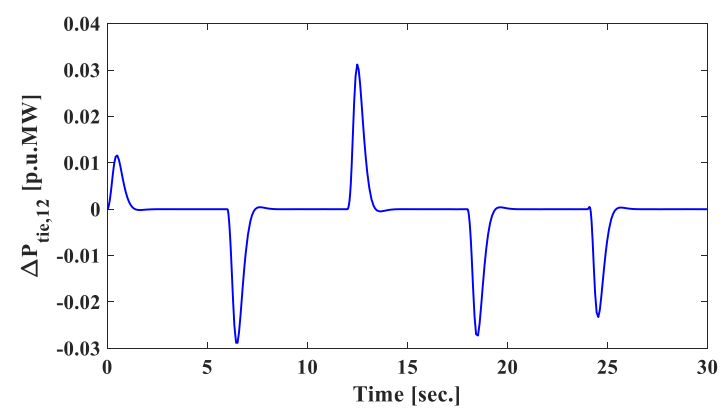

Fig. 6: Tie-line variation.

Remark 5: The powerful of integral SMC is examined against via random load disturbances. The performance of system is better in term of faster settling time and smaller overshoot. It also demonstrates that the suggested controller can be applied to the real power network with communication delay.

Case 3: In this case, the effectiveness of the suggested method is examined with different conditions including the step load disturbance and the mismatched uncertainty. In which, the values of step load disturbances are assumed as $\Delta P_{d 1}(t)=0.015$ at $1 \mathrm{~s}$ in the first area and $\Delta P_{d 2}(t)=0.005$ at $1 \mathrm{~s}$ in the second area. To examine robustness of the proposed SMC controller, the mismatched parameter uncertainty of TAPN is selected with value the same with [38] and is rewritten as $\Delta A$ (see on the next page).

In cases 1 and 2, the effect of GRC is not mentioned. In this case, the GRC limiter is considered for the dynamic responses of the TAPS. The open loop modeling of GRC is displayed in Fig. 7 for the thermal power station and Fig. 8 for hydro power stations [40]. The responses of the suggested method for the TAPN with GRC of $0.01 \mathrm{pu} / \mathrm{min}$, or $0.0017 \mathrm{pu} / \mathrm{sec}$ are displayed in Figs. 9-11. In which, Figs. 9 and 10 represent frequency variation $\left(\Delta f_{1}, \Delta f_{2}\right)$ in both areas. We easily observe that the system frequency is kept in nominal value due to there is no change in load at the initial time. After one second, with the sudden change of load, the frequency deviation is changed from the desired value to the maximum deviation as $-0.014 \mathrm{pu}$ of area 1 and $-0.011 \mathrm{pu}$ of area 2. Then, the proposed controller starts operating and controlling valve position to adjust steam/water into the turbine for balancing the generator power and load power. Fig. 10 displays the deviation of tie-line power.

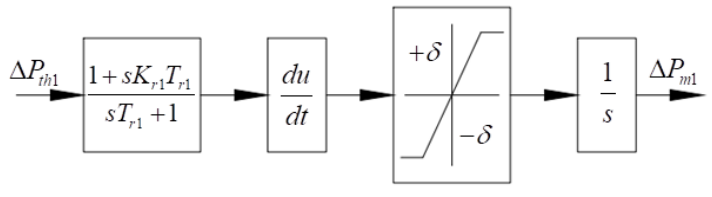

Fig. 7: Open loop modeling of thermal turbine with GRC.

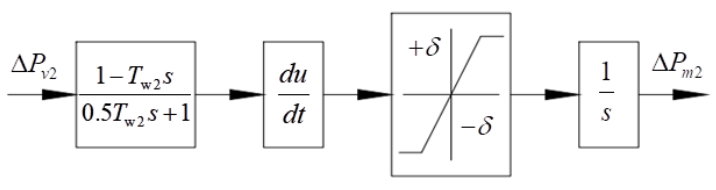

Fig. 8: Open loop modeling of hydro turbine with GRC.

The GRC impacts significantly the performance of the ITDPS. Observing the simulation results of three cases, we easily realize that the settling time is longer in case of considering the GRC than other cases. Hence, system performance is 
00000000000

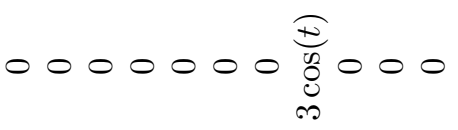

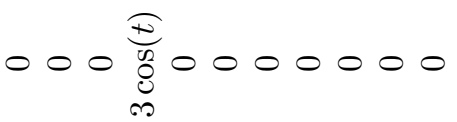

00000000000

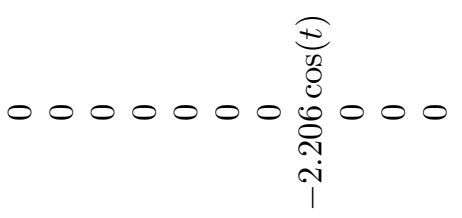

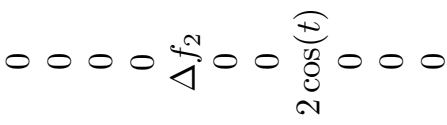

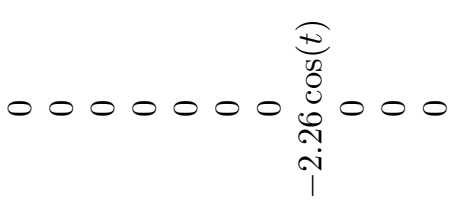

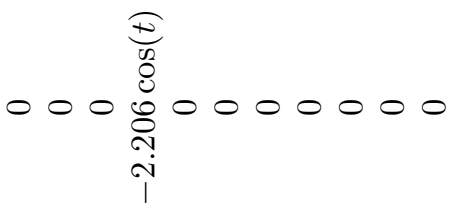

00000000000

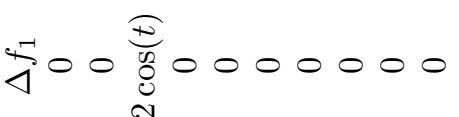

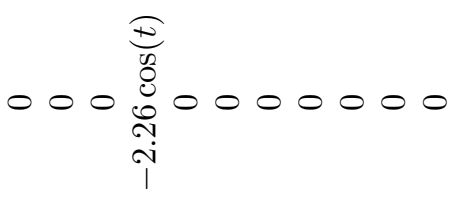

$\stackrel{\square}{\square}$

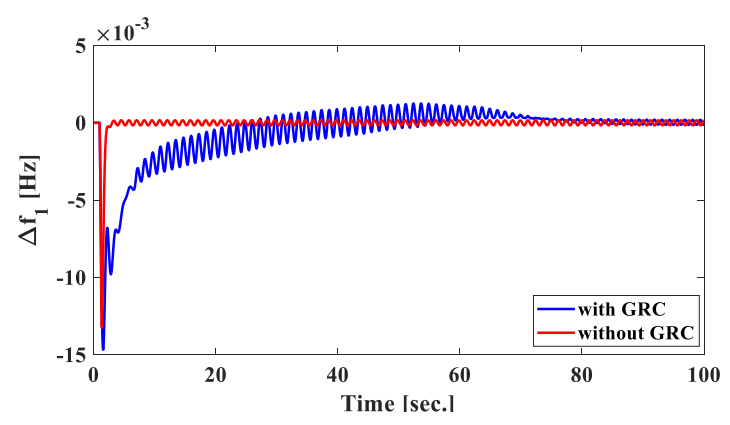

Fig. 9: Frequency variation of the $1^{\text {st }}$ area.

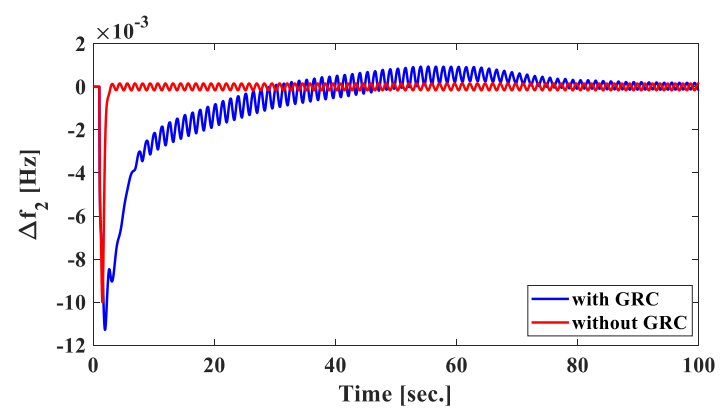

Fig. 10: Frequency variation of the $2^{\text {nd }}$ area.

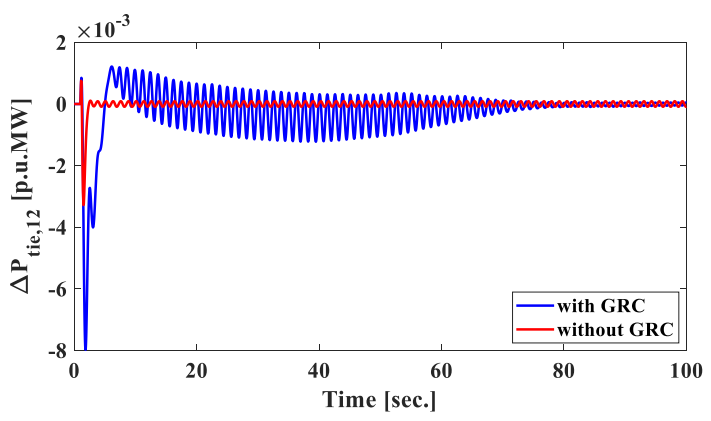

Fig. 11: Tie-line variation.

better without the GRC. Fig. 9 and Fig. 10 also show that the suggested controller can make the system stable with GRC.

Remark 6: The amplitude of the frequency deviation $\Delta f_{1}$ always greater than amplitude of the frequency deviation $\Delta f_{2}$ because the changing of the power output of the thermal power station in the area 1 is slower than the changing of the power output of the hydro power station in the area 2. 
Remark 7: The simulation results prove that the suggested method is robust and can prevent the system from the affection of mismatched parameter uncertainty and communication delay. However, there are still small oscillations around equilibrium point. This is the need of the research in the future by applying higher-order $S M C$.

\section{Conclusions}

In this paper, we present a sliding mode control for ITDPS with two source power generations to solve the LFC problem. The suggested controller is implemented by the SMC method. The stability of the ITDPS is guaranteed via the LMI technique which is derived based on Lyapunov stability theory. The experimental simulations are applied for the two-area power network with thermal and hydro power stations under different value of the time delay for each area. The controller action effectively adjusts the power generation of two areas to minimize tie-line power flows and frequency error deviation.

At present, due to the difficulty in measurement of the system state of the ITDPS, the combination of observer and higher order SMC for the LFC of the ITDPS has always been a topic of concern, which motivates us to carry out in the future.

\section{Appendix A}

$K_{B 1}, K_{B 2}$ - Frequency bias constant of $i$ th control area, pu $\mathrm{MW} / \mathrm{Hz}$

$T_{g 1}, T_{g 2}$ - Speed governor time constant, $\mathrm{s}$

$T_{t h 1}$ - Steam turbine time constant, $\mathrm{s}$

$T_{p 1}, T_{p 2}$ - Power system time constant, $\mathrm{s}$

$K_{p 1}, K_{p 2}$ - Power system gain, $\mathrm{Hz} / \mathrm{pu} \mathrm{MW}$

$K_{r 1}$ - Steam turbine reheats constant

$T_{r 1}$ - Steam turbine reheats time constant, $\mathrm{s}$

$T_{\mathrm{w} 2}$ - Nominal starting time of water in penstock, s
$T_{r s 2}-$ Hydro turbine speed governor reset time, $\mathrm{s}$

$T_{r h 2}$ - Hydro turbine speed governor transient droop time constant, $\mathrm{s}$

$T_{12}$ - Tie line power coefficient

$\Delta f_{1}, \Delta f_{2}$ - Incremental change in frequency of $i$ th control area, $\mathrm{Hz}$

$\Delta P_{t i e 12}$ - Incremental change in actual tie line power flow from control area-1 to 2

$\Delta P_{d 1}, \Delta P_{d 2}-$ Total incremental change in local load of $i$ th control area, pu MW

$\Delta \mathrm{E}_{1}, \Delta \mathrm{E}_{2}-$ Area control error of $i$ th control area, pu MW

$\Delta P_{m 1}, \Delta P_{m 2}-$ Incremental change in power output of ith generating unit, pu MW

$\Delta P_{\text {ref1 }}, \Delta P_{\text {ref2 }}-$ Incremental change in speed changer position of $i$ th of area, pu MW

$\Delta P_{g 2}$ - Incremental change in governor output command, pu MW

$\Delta P_{t h 1}-$ Incremental change steam turbine, pu MW

$\Delta P_{v 1}, \Delta P_{v 2}-$ Incremental change in governor valve position, pu MW.

\section{Appendix B}

General $K_{P 1}=K_{P 2}=65.2174 \mathrm{~Hz} / \mathrm{pu} \mathrm{MW}$; $T_{P 1}=T_{P 2}=10.8696 \mathrm{~s} ; K_{E 1}=K_{E 2}=1$; $T_{12}=0.0433 ; K_{B 1}=K_{B 2}=1 ; R_{1}=R_{2}=2.4$ $\mathrm{Hz} / \mathrm{pu} \mathrm{MW}$;

Reheat Turbine $T_{t h 1}=0.08 \mathrm{~s} ; K_{R 1}=0.3 ; T_{r 1}=$ $10 \mathrm{~s} ; T_{g 1}=0.3 \mathrm{~s}$;

Hydro Turbine $T_{g 2}=0.2 \mathrm{~s} ; T_{r s 2}=5 \mathrm{~s} ; T_{r h 2}=$ $28.75 \mathrm{~s}$;

\section{References}

[1] Saxena, S., \& Hote, Y.V. (2016). Decentralized PID load frequency control for perturbed multi-area power systems. International Journal of Electrical Power $\& 3$ Energy Systems, 81, 405-415. 
[2] Tan, W., \& Zhou, H. (2012). Robust analysis of decentralized load frequency control for multi-area power systems. International Journal of Electrical Power 83 Energy Systems, 43(1), 996-1005.

[3] Singh, V.P., Mohanty, S.R., Kishor, N., \& Ray, P.K. (2013). Robust H-infinity load frequency control in hybrid distributed generation system. International journal of electrical power 83 energy systems, 46, 294305.

[4] Yang, T.C., \& Cimen, H. (1996). Applying structured singular values and a new LQR design to robust decentralized power system load frequency control. Proceedings of the IEEE International Conference on Industrial Technology (ICIT'96), 880-884.

[5] Sivaramakrlshnan, A.Y., Hariharan, M.V., \& Srisailam, M.C. (1984). Design of variable-structure load-frequency controller using pole assignment technique. International Journal of control, 40(3), 487-498.

[6] Tan, W. (2009). Unified tuning of PID load frequency controller for power systems via IMC. IEEE Transactions on power systems, $25(1), 341-350$.

[7] Singh, V.P., Kishor, N., \& Samuel, P. (2017). Improved load frequency control of power system using LMI based PID approach. Journal of the Franklin Institute, $354(15), 6805-6830$.

[8] Shabani, H., Vahidi, B., \& Ebrahimpour, M. (2013). A robust PID controller based on imperialist competitive algorithm for load-frequency control of power systems. ISA transactions, 52(1), 88-95.

[9] Rajesh, K.S., \& Dash, S.S. (2019). Load frequency control of autonomous power system using adaptive fuzzy based PID controller optimized on improved sine cosine algorithm. Journal of Ambient Intelligence and Humanized Computing, 10(6), 23612373 .

[10] Zamani, A., Barakati, S.M., \& YousofiDarmian, S. (2016). Design of a fractional order PID controller using GBMO algorithm for load-frequency control with governor saturation consideration. ISA transactions, 64, 56-66.

[11] Sondhi, S., \& Hote, Y.V. (2014). Fractional order PID controller for load frequency control. Energy Conversion and Management, 85, 343-353.

[12] Abdelaziz, A.Y., \& Ali, E.S. (2016). Load frequency controller design via artificial cuckoo search algorithm. Electric Power Components and Systems, 44(1), 90-98.

[13] Gautam, S.K., \& Goyal, N. (2010). Improved particle swarm optimization based load frequency control in a single area power system. 2010 Annual IEEE India Conference (INDICON), 1-4.

[14] Zribi, M., Al-Rashed, M., \& Alrifai, M. (2005). Adaptive decentralized load frequency control of multi-area power systems. International Journal of Electrical Power \& Energy Systems, 27(8), 575-583.

[15] Yousef, H.A., Khalfan, A.K., Albadi, M.H., \& Hosseinzadeh, N. (2014). Load frequency control of a multi-area power system: An adaptive fuzzy logic approach. IEEE transactions on power systems, 29(4), 18221830.

[16] Maher, R.A., Mohammed, I.A., \& Ibraheem, I.K. (2014). Polynomial based $\mathrm{H} \infty$ robust governor for load frequency control in steam turbine power systems. International Journal of Electrical Power \& Energy Systems, 57, 311-317.

[17] Toulabi, M.R., Shiroei, M., \& Ranjbar, A.M. (2014). Robust analysis and design of power system load frequency control using the Kharitonov's theorem. International Journal of Electrical Power 83 Energy Systems, 55, 51-58.

[18] Orlowska-Kowalska, T., Tarchala, G., \& Dybkowski, M. (2014). Sliding-mode direct torque control and sliding-mode observer with a magnetizing reactance estimator for the field-weakening of the induction motor 
drive. Mathematics and Computers in Simulation, 98, 31-45.

[19] Dou, C., Zhang, B., Yue, D., Zhang, Z., Xu, S., Hayat, T., \& Alsaedi, A. (2018). A novel hierarchical control strategy combined with sliding mode control and consensus control for islanded micro-grid. IET Renewable Power Generation, 12(9), 10121024.

[20] Alsmadi, Y.M., Utkin, V., Haj-ahmed, M.A., \& Xu, L. (2018). Sliding mode control of power converters: DC/DC converters. International Journal of Control, $91(11), 2472-2493$.

[21] Nguyen, S.D., \& Nguyen, Q.H. (2017). Design of active suspension controller for train cars based on sliding mode control, uncertainty observer and neuro-fuzzy system. Journal of Vibration and Control, 23(8), 1334-1353.

[22] Nguyen, S.D., \& Lam, B.D. (2020). Fractional-order sliding-mode controller for semi-active vehicle MRD suspensions. Nonlinear Dynamics, 101(2), 795-821.

[23] Tran, A.T., Minh, B.L.N., Huynh, V.V., Tran, P.T., Amaefule, E.N., Phan, V. D., \& Nguyen, T. M. (2021). Load Frequency Regulator in Interconnected Power System Using Second-Order Sliding Mode Control Combined with State Estimator. Energies, $14(4), 863$.

[24] Huynh, V.V., Minh, B.L.N., Amaefule, E.N., Tran, A.T., \& Tran, P.T. (2021). Highly Robust Observer Sliding Mode Based Frequency Control for Multi Area Power Systems with Renewable Power Plants. Electronics, 10(3), 274.

[25] Xu, J.X., Guo, Z.Q., \& Lee, T.H. (2013). Design and implementation of integral sliding-mode control on an underactuated two-wheeled mobile robot. IEEE Transactions on industrial electronics, 61(7), 36713681 .
[26] Mi, Y., Yang, Y., Zhang, H., Yu, A., Wang, L., \& Ren, L. (2014). Sliding mode based load frequency control for multi-area interconnected power system containing renewable energy. 2014 IEEE Conference and Expo Transportation Electrification AsiaPacific (ITEC Asia-Pacific), 1-6.

[27] Le Ngoc Minh, B., Huynh, V.V., Nguyen, T.M., \& Tsai, Y.W. (2018). Decentralized adaptive double integral sliding mode controller for multi-area power systems. Mathematical Problems in Engineering.

[28] Mu, C., Tang, Y., \& He, H. (2017). Improved sliding mode design for load frequency control of power system integrated an adaptive learning strategy. IEEE Transactions on Industrial Electronics, 64(8), 6742-6751.

[29] Guo, X., \& Liu, X. (2014). Particle swarm optimization sliding mode control on interconnected power system. IEEE Proceedings of the 33rd Chinese Control Conference, 9397.

[30] Mi, Y., Hao, X., Liu, Y., Fu, Y., Wang, C., Wang, P., \& Loh, P.C. (2017). Sliding mode load frequency control for multi-area time-delay power system with wind power integration. IET Generation, Transmission E Distribution, 11 (18), 4644-4653.

[31] Onyeka, A.E., Xing-Gang, Y., Mao, Z., Jiang, B., \& Zhang, Q. (2019). Robust decentralised load frequency control for interconnected time delay power systems using sliding mode techniques. IET Control Theory \& Applications, $14(3), 470-480$.

[32] Sun, Y., Wang, Y., Wei, Z., Sun, G., \& Wu, X. (2017). Robust $\mathrm{H} \infty$ load frequency control of multi-area power system with time delay: a sliding mode control approach. IEEE/CAA Journal of Automatica Sinica, $5(2), 610-617$. 
[33] Boyd, S., El Ghaoui, L., Feron, E., \& Balakrishnan, V. (1994). Linear matrix inequalities in system and control theory. Society for industrial and applied mathematics.

[34] Huynh, V.V., Tsai, Y.W., \& Duc, P. V. (2015). Adaptive output feedback sliding mode control for complex interconnected time-delay systems. Mathematical Problems in Engineering.

[35] Yan, X.G., Spurgeon, S.K., \& Edwards, C. (2012). Global decentralised static output feedback sliding-mode control for interconnected time-delay systems. IET control theory $\&$ applications, 6(2), 192-202.

[36] Guo, J. (2019). Application of full order sliding mode control based on different areas power system with load frequency control. ISA transactions, 92, 23-34.

[37] Manikandan. S., \& Priyanka Kokil (2020). Stability Analysis of Load Frequency Control System with Constant Communication Delays. International Federation of Automatic Control, 53(1), 338-343.

[38] Tran, A.T., Le Ngoc Minh, B., Tran, P.T., Huynh, V.V., Phan, V.D., Pham, V.T., \& Nguyen, T.M. (2021). Adaptive Integral Second-Order Sliding Mode Control Design for Load Frequency Control of LargeScale Power System with Communication Delays. Complexity.

[39] Tran, A.T., Tran, P.T., \& Van Huynh, V. (2021). Load Frequency Control for Power System using Generalized Extended State Observer. Journal of Advanced Engineering and Computation, 5(1), 1-18.

[40] Nikmanesh, E., Hariri, O., Shams, H., \& Fasihozaman, M. (2016). Pareto design of Load Frequency Control for interconnected power systems based on multiobjective uniform diversity genetic algorithm (MUGA). Electrical Power and Energy Systems, 80, 333-346.

\section{About Authors}

Bao Hoan LAI received B.Eng. degree in Automation and Control Enginering, Ton Duc Thang University (TDTU), Ho Chi Minh City, Vietnam. Currently, he is M.Eng. student in Automation and Control Engineering, TDTU, HCMC, Vietnam. His main research topics included load frequency control for power systems with time delays, power system stability and control, and applications to process power systems.

Anh-Tuan TRAN received B.Eng. degree in electrical engineering, Ton Duc Thang University (TDTU), Ho Chi Minh City, Vietnam. Currently, he is M.Eng. student in Electrical Engineering, TDTU, HCMC, Vietnam. His research topics interests are in load frequency control for power systems with sliding mode control.

Van Van HUYNH has completed the Ph.D degree in automation and control from Da-Yeh University, Taiwan. He is currently a Lecturer in the Faculty of Electrical and Electronics Engineering, Ton Duc Thang University, Ho Chi Minh City, Vietnam. He has published totally 25 journal papers and more than 11 international conference papers. His current research interests are in sliding mode control, variable structure control, and power system control.

Nhat Truong PHAM received a B.Eng. egree in Electronics and Telecommunication Engineering, Ton Duc Thang University (TDTU), Vietnam, in 2019. He is currently pursuing an M.Eng. degree in Automation and Control Engineering. $\mathrm{He}$ is a research assistant in the Division of Computational Mechatronics, Institute for Computational Science, TDTU, Vietnam. His research interests include Artificial Intelligence, Deep Learning, Computer Vision, Signal Processing, Robotics, and Intelligent Computation.

"This is an Open Access article distributed under the terms of the Creative Commons Attribution License, 107 which permits unrestricted use, distribution, and reproduction in any medium provided the original work is properly cited (CC BY 4.0)." 